\title{
Smooth and rough boundaries in turbulent Taylor-Couette flow
}

\author{
Thomas H. van den Berg, ${ }^{1,4}$ Charles R. Doering, ${ }^{2,3}$ Detlef Lohse, ${ }^{1}$ and Daniel P. Lathrop ${ }^{4}$ \\ ${ }^{1}$ Department of Applied Physics and J. M. Burgers Centre for Fluid Dynamics, University of Twente, P.O. Box 217, 7500 AE Enschede, \\ The Netherlands \\ ${ }^{2}$ Department of Mathematics, University of Michigan, Ann Arbor, Michigan 48109-1109, USA \\ ${ }^{3}$ Michigan Center for Theoretical Physics, Ann Arbor, Michigan 48109-1120, USA \\ ${ }^{4}$ Department of Physics, IREAP and IPST, University of Maryland, College Park, Maryland 20742, USA
}

(Received 31 January 2003; published 12 September 2003)

\begin{abstract}
We examine the torque required to drive the smooth or rough cylinders in turbulent Taylor-Couette flow. With rough inner and outer walls the scaling of the dimensionless torque $G$ is found to be consistent with pure Kolmogorov scaling $G \sim \operatorname{Re}^{2}$. The results are interpreted within the Grossmann-Lohse theory for the relative role of the energy dissipation rates in the boundary layers and in the bulk; as the boundary layers are destroyed through the wall roughness, the torque scaling is due only to the bulk contribution. For the case of one rough and one smooth wall, we find that the smooth cylinder dominates the dissipation rate scaling, i.e., there are corrections to Kolmogorov scaling. A simple model based on an analogy to electrical circuits is advanced as a phenomenological organization of the observed relative drag functional forms. This model leads to a qualitative prediction for the mean velocity profile within the bulk of the flow.
\end{abstract}

DOI: 10.1103/PhysRevE.68.036307

The relation between global transport properties and the external driving forces is the focus of interest for various turbulent flows. Examples include Rayleigh-Bénard convection where the heat flux is studied as a function of the temperature difference between top and bottom walls, pipe flow where the mean flow is studied as a function of the pressure drop, and Taylor-Couette flow where the required torque is studied as a function of the cylinder rotation speed. For many of these examples strict upper bounds exist (see, e.g., Ref. [1] for Rayleigh-Benard flow or Ref. [2] for Taylor-Couette flow), but no exact calculation yields the flux-forcing dependence.

To better understand the relation between the global transport properties and the driving forces, it is important to examine the role of the flow structure and boundary conditions. For Rayleigh-Bénard convection this has been done by employing rough boundary conditions [3-9] or by varying the aspect ratio or the Prandtl number [10-12]. Also for pipe flow the effect of rough boundary layers on the global transport efficiency has long been studied and parametrized in the roughness dependence of the skin friction coefficient $[13,14]$.

For Taylor-Couette flow the effect of two rough walls has been examined by Cadot et al. [15]. Their main finding is that the total energy dissipation rate $\epsilon$ scales as

$$
\epsilon \sim \frac{U^{3}}{(b-a)},
$$

where $b$ is the outer radius, $a$ is the inner radius, and $U$ is the velocity of the inner cylinder. This rough-wall result is consistent with Kolmogorov's expectation of a residual dissipation at zero viscosity for fully developed turbulence [16], but in contrast to the smooth-wall result, where corrections to Eq. (1) are known to occur [17-19].

The aim of the present study is to extend the results by Cadot $e$ al. to the situation when only one of the cylinders is rough. An informal survey of some knowledgeable colleagues showed a lack of consensus on the answer to the
PACS number(s): 47.27.Nz, 47.27.Jv, 47.27.Pa

following question: which boundary layer dominates the scaling, i.e., would the dissipation dependence show corrections to the Kolmogorov expectation (1) for one rough and one smooth wall? The analysis in Ref. [20] shows that the turbulent Kolmogorov scaling in Eq. (1) is an upper bound for smooth walls, but it can also be extended to prove that it is also a rigorous upper limit on $\epsilon$ for one rough and one smooth wall [21]. Hence this experimental question is also of interest to interpret the upper bound results.

One argument considered prior to our empirical results involved the fluctuations. The level of velocity fluctuations in the system might be set by the boundary generating the largest disturbances. The resulting fluctuations would effect Reynolds stresses which would dominate the momentum transport, and therefore the dissipation. The rough wall would generate the largest level of the fluctuations, so this could be used to argue that it dominates and no logarithmic corrections might result.

That view turns out to be empirically incorrect, which may be understood using the Grossmann-Lohse theory for the relative role of energy dissipation rate into the boundary layers and in the bulk. This theory has not only been developed for Rayleigh-Bénard convection [22-24], but also applied to Taylor-Couette and pipe flow [25]. The central idea is to split the total energy dissipation rate $\epsilon$ (and for the Rayleigh-Bénard case also the thermal dissipation rate) into a boundary layer (BL) and a bulk contribution,

$$
\epsilon=\epsilon_{B L}+\epsilon_{\text {bulk }} .
$$

The total energy dissipation rate $\epsilon$ in Taylor-Couette flow can be rigorously related with the dimensionless torque $G$, namely [19],

$$
\epsilon=\frac{\nu^{2} G \Omega}{2 \pi\left(b^{2}-a^{2}\right)}
$$

Here $G=T / \rho \nu^{2} L$ is the dimensionless torque, $T$ the torque, $\rho$ the fluid density, $\nu$ its kinematic viscosity, $L$ the length of 
the cylinders, and $\Omega$ the angular rotation rate. The Reynolds number $\operatorname{Re}$ is defined as $\operatorname{Re}=\Omega a(b-a) / \nu$. Following $\operatorname{Ref}$. [22], the energy dissipation rate in the bulk is estimated as

$$
\epsilon_{b u l k} \sim \frac{U^{3}}{(b-a)}
$$

and that in the BL as

$$
\epsilon_{B L} \sim \nu \frac{U^{2}}{\lambda_{u}^{2}} \frac{\lambda_{u}}{b-a},
$$

where $\lambda_{u} \sim(b-a) / \sqrt{\operatorname{Re}}$ is the thickness of a laminar boundary layer assumed to be of Blasius-Prandtl type. ${ }^{1}$ With these two estimates we obtain

$$
G=c_{1} \operatorname{Re}^{3 / 2}+c_{2} \operatorname{Re}^{2} .
$$

The first term originates from contribution of the laminar boundary layers, the second one from that of the bulk. For the smooth-wall case, the Grossmann-Lohse theory accounts for the Rayleigh Ra (and Prandtl) number dependence of the experimentally found Nusselt $\mathrm{Nu}$ (and Reynolds) number for the Rayleigh-Bénard case. For the Taylor-Couette case, it gives the Reynolds number dependence of the dimensionless torque $G$.

How do these considerations in convection extend to the rough-wall cases? In Rayleigh-Bénard flow, for large enough roughness and $\mathrm{Ra}$, the laminar boundary layers are expected to break down and become turbulent. This results in the ultimate scaling regime, $\mathrm{Nu} \sim \mathrm{Ra}^{1 / 2} \operatorname{Pr}^{1 / 2}$, in which the total dissipation rate scales solely with the dissipation rate in the bulk, due to the lack of laminar boundary layers [22]. The scaling exponent $1 / 2$ in the Ra-Nu dependence had been predicted earlier $[26,27]$ for thermal convection. The $1 / 2$ scaling exponent is also found [28] in numerical simulations in which the boundary layers have been eliminated and replaced by periodic boundary conditions. Rough-wall experiments on Rayleigh-Benard convection by Roche et al. [9] also find a transition towards the $\mathrm{Nu} \sim \mathrm{Ra}^{1 / 2}$ scaling law, the upper bound result [1].

For the rough-wall Taylor-Couette experiment the corresponding expectation is $G \sim \mathrm{Re}^{2}$ for large enough roughness or Reynolds number, yielding again the upper bound result [2]. This has been observed experimentally by Cadot et al. [15]. We expect the boundary layer influence to become relatively smaller when one of the walls is roughened. That is, the ratio of the bulk to boundary layer contributions to the energy dissipation rate should increase and this is observed in the data.

The experimental apparatus consists of a rotating inner cylinder of radius $a=16 \mathrm{~cm}$, and a stationary outer cylinder

\footnotetext{
${ }^{1}$ Reference [25] distinguishes between the imposed velocity $U$ and the typical velocity difference $U_{w}$ between the turbulent and the laminar (linear) profiles, in order to better describe the experimental data. There a power law relation $U_{w} / U \sim \operatorname{Re}^{\xi}$ was assumed, with a best fit of $\xi=-0.051$. Here, we drop this distinction and assume $U \sim U_{w}$, for simplicity and as $\xi=0$ seems to satisfactorily describe the present data.
}
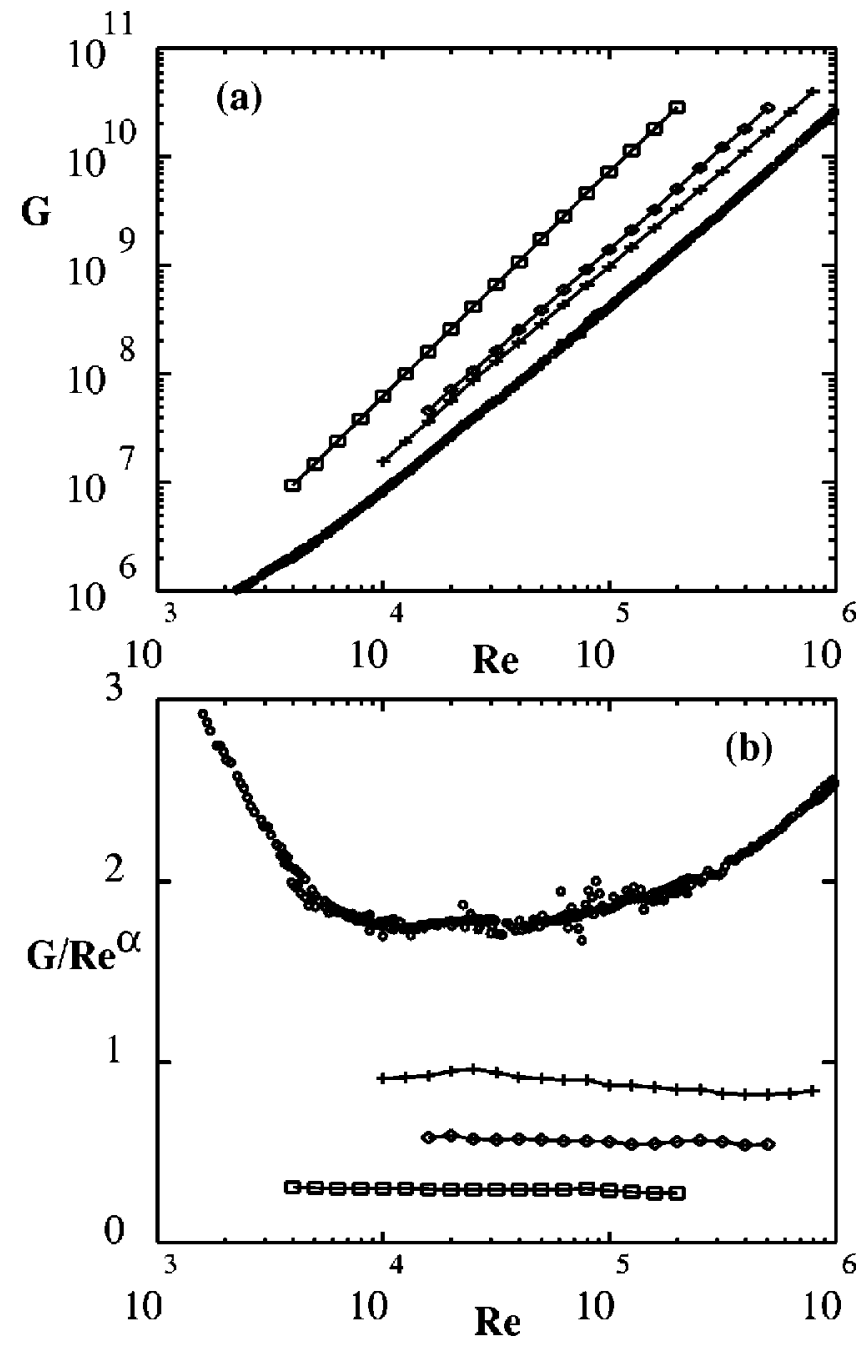

FIG. 1. (a) The dimensionless torque $G=T / \rho \nu^{2} L$ shows a dependence on Reynolds number [dimensionless forcing $\operatorname{Re}=\Omega a(b$ $-a) / \nu$ ] close to Kolmogorov scaling $G \sim \operatorname{Re}^{2}$. The four cases shown differ on the condition of the cylinders: $\bigcirc$ (ss) both cylinders smooth; + (sr) smooth inner, rough outer; $\diamond$ (rs) rough inner, smooth outer; and $\square$ (rr) both walls rough. The deviations from that scaling indicate the importance and structure of the boundary layers. (b) Compensated plots $G / \operatorname{Re}^{\alpha}$ dependence on Reynolds number, using the values $\alpha$ from Table I.

of radius $b=22 \mathrm{~cm}$, yielding a radius ratio of $\eta=a / b$ $=0.73$. The height of the gap was $L=69.5 \mathrm{~cm}$, giving an aspect ratio of $\Gamma=L /(b-a)=12$. The inner cylinder was especially instrumented to measure the torque on a $40.64 \mathrm{~cm}$ central section using strain gauges. This apparatus is described in more detail in Refs. $[17,19]$. Our measurements span a Reynolds number, $\operatorname{Re}=\Omega a(b-a) / \nu$, range of $10^{4}$ $<\operatorname{Re}<10^{6}$, where $\Omega$ is the inner cylinder rotation rate. Three fluids were used: water and water-glycerin mixtures of viscosities $\nu=0.01,0.10$, and $0.26 \mathrm{~cm}^{2} / \mathrm{s}$, respectively. Either the inner, the outer, or both of the cylinders were roughened by attaching 16 vertical strips of square cross section (0.3 cm on edge) which were equally spaced in azimuthal angle, similar to the procedure used in Ref. [15].

Four cases are analyzed: (ss) smooth inner and outer wall, 
TABLE I. Results from a power law fit $G=c \operatorname{Re}^{\alpha}$. In the last line we have fixed the exponent $\alpha=2$.

\begin{tabular}{ccc}
\hline \hline Case & $c$ & $\alpha$ \\
\hline ss & 2.14 & 1.67 \\
sr & 0.79 & 1.81 \\
rs & 0.48 & 1.88 \\
rr & 0.21 & 2.08 \\
rr & 0.57 & 2 \\
\hline
\end{tabular}

(sr) smooth inner and rough outer wall, (rs) rough inner and smooth outer wall, and (rr) rough inner and outer wall. The maximum rotation rate achieved in each case was determined approximately by the available motor torque.

Figure 1(a) shows $G$ vs $\operatorname{Re}$ for the four cases. Fitting a power law

$$
G=c \operatorname{Re}^{\alpha}
$$

gives the exponents shown in Table I. It is clearly seen that the approximate power law exponent $\alpha$ increases with the roughness of the walls and is consistent with $\alpha=2$ in the rr case.

The alternative way to represent the data is that according to the Grossmann-Lohse theory, namely, $G / \operatorname{Re}^{3 / 2}$ vs $\operatorname{Re}^{1 / 2}$, see Fig. 2. From a linear regression to those data the coefficients $c_{1}$ and $c_{2}$ of Eq. (6) can directly be obtained, see Table II. The expectation discussed above that ratio of the laminar BL contribution $c_{1} \mathrm{Re}^{3 / 2}$ and the turbulent bulk contribution $c_{2} \mathrm{Re}^{2}$ becomes less when one wall is roughened and becomes close to zero when both walls are roughened is observed. As for the rr case the laminar BLs should be com-

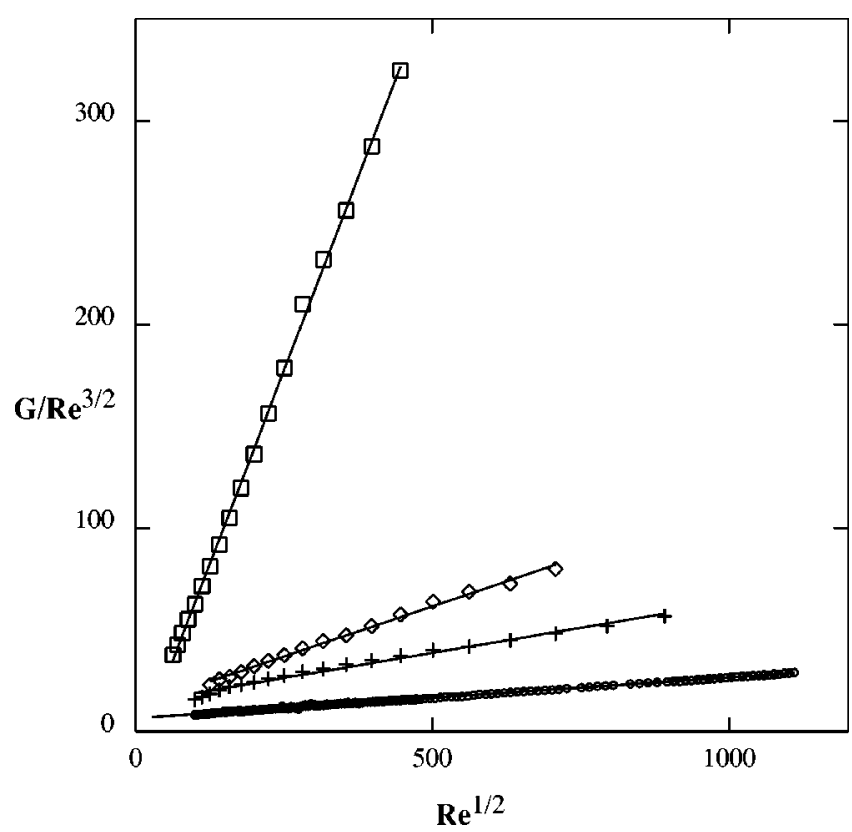

FIG. 2. A test of the scaling prediction Eq. (6); $G / \operatorname{Re}^{3 / 2} \mathrm{vs} \mathrm{Re}^{1 / 2}$ for the four cases $(\bigcirc) \mathrm{ss},(+) \mathrm{sr},(\diamond) \mathrm{rs}$, and $(\square) \mathrm{rr}$, bottom to top. A linear regression to these data gives the coefficients $c_{1}$ and $c_{2}$ in Eq. (6) as shown in Table II.
TABLE II. Results from a linear regression to the experimental data of $G / \operatorname{Re}^{3 / 2}=c_{1}+c_{2} \operatorname{Re}^{1 / 2}$. In the last line we have enforced $c_{1}=0$. In the second but last line there is hardly any contribution from the laminar BL, so $c_{1}$ gets an ill-defined value.

\begin{tabular}{cccc}
\hline \hline Case & $c_{1}$ & $c_{2}$ & $\epsilon_{\text {bulk }}\left(10^{5}\right) / \epsilon_{B L}\left(10^{5}\right)$ \\
\hline $\mathrm{ss}$ & 6.69 & 0.0201 & 0.95 \\
$\mathrm{sr}$ & 14.1 & 0.0493 & 1.11 \\
$\mathrm{rs}$ & 12.4 & 0.100 & 2.55 \\
$\mathrm{rr}$ & -12.8 & 0.76 & -18.8 \\
$\mathrm{rr}^{\prime}$ & 0 & 0.571 & $\infty$ \\
\hline \hline
\end{tabular}
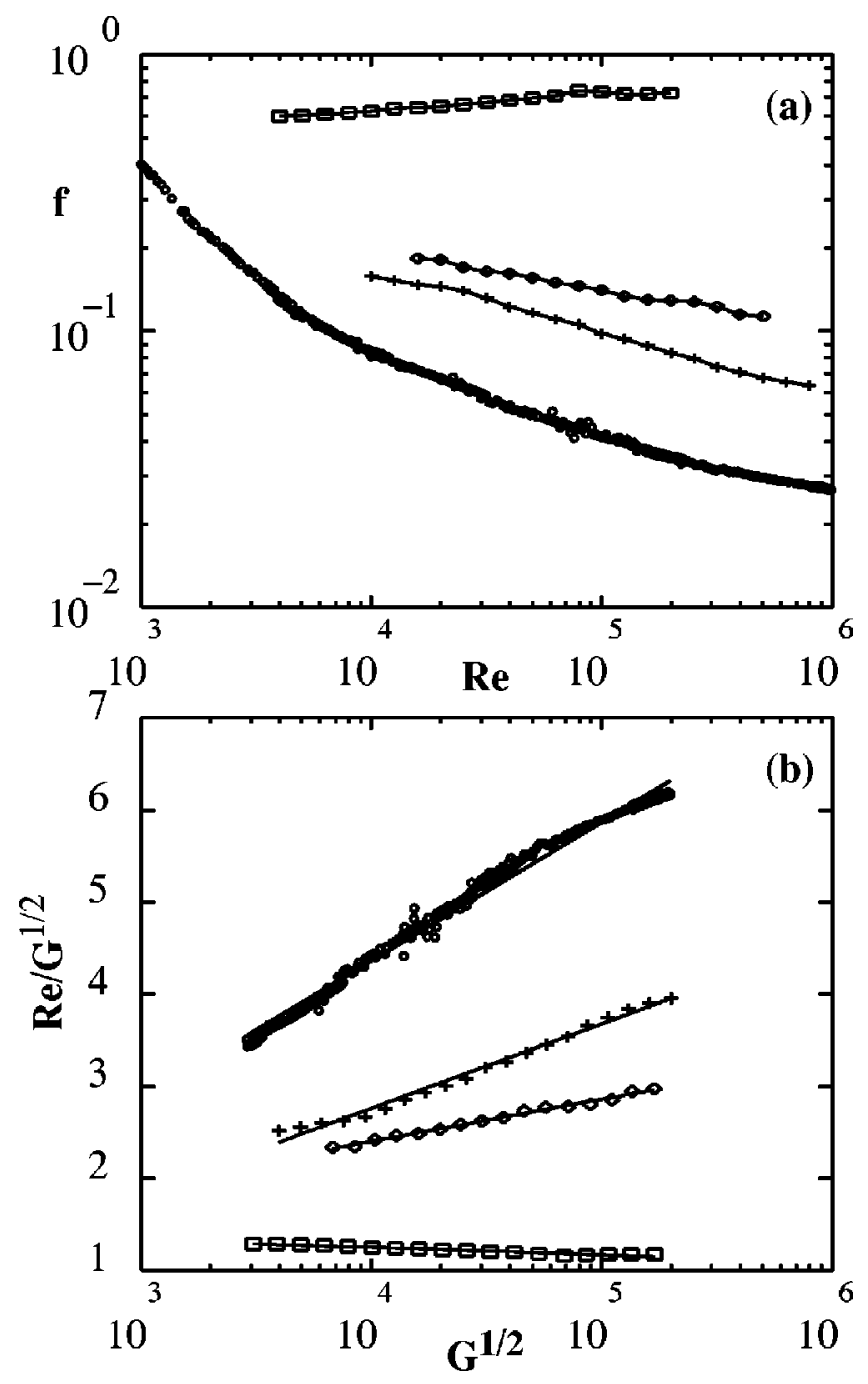

FIG. 3. (a) Skin friction coefficient $f$ vs Reynolds number Re for the four cases $(\bigcirc) \mathrm{ss},(+) \mathrm{sr},(\diamond) \mathrm{rs}$, and $(\square) \mathrm{rr}$, bottom to top. For the ss case the data are well described by the skin friction law (8) (solid line, see also Table III), as extensively discussed in Refs. [17-19]. In the rr case, $f$ becomes independent of Re for large enough Re. The cases rs and sr with one rough wall only are in between these extreme cases. Due to the persistence of one smooth wall and the corresponding boundary layer, $f$ still depends on Re for large Re. (b) Same data as in (a), but now plotted as $1 / \sqrt{f}$ $=\operatorname{Re} / \sqrt{G}$ vs $\log _{10}(\operatorname{Re} \sqrt{f})=\log _{10} G^{1 / 2}$. If Eq. (8) is right, this type of plot results in a straight line. 
TABLE III. Results from fitting the Prandtl-von Kármán friction law (8) to the experimental data. The cases with rough walls have a poor correlation coefficient, as this model is least suitable for that case.

\begin{tabular}{cccc}
\hline \hline Case & $c_{1}^{\prime}$ & $c_{2}^{\prime}$ & Correlation coefficient \\
\hline ss & 1.51 & -1.66 & 0.99 \\
sr & 0.920 & -0.917 & 0.99 \\
rs & 0.457 & 0.575 & 0.99 \\
rr & -0.0792 & 1.57 & 0.95 \\
\hline \hline
\end{tabular}

pletely vanished for large enough Re number, we also tried the one-parameter fit $G=c_{2} \operatorname{Re}^{2}$ which well describes the data.

We also test the Prandtl-von Kármán skin friction law for this data. For the friction coefficient $f=G / \mathrm{Re}^{2}$ it holds for smooth boundaries $[13,18,19,25]$

$$
\frac{1}{\sqrt{f}}=c_{1}^{\prime} \log _{10}(\operatorname{Re} \sqrt{f})+c_{2}^{\prime} \text {. }
$$

This friction law (8) well fits the data for the ss case (see Fig. 3 ). For the case with rough walls a roughness length scale must be introduced, see Sec. 7.2 of Ref. [14]. For large enough roughness and large enough Re the skin friction coefficient $f$ then becomes independent of the Reynolds number, as indeed observed from Fig. 3(a).

How can one rationalize the observed dependences of the torques in the four different cases (rr, sr, rs, and ss)? We have attempted to do so by employing a circuit analogy. Although this analogy is imperfect, we hope this will stimulate more progress on the special problems associated with situations where the momentum must flow through several subcomponents in series.

The torque, and therefore dissipation, is determined by the flux of angular momentum transported radially through the fluid from the inner cylinder to the outer cylinder. The conserved quantity is the torque, i.e., the torque on both cylinders-or through any concentric cylinder in between-is the same. This is analogous to the current in a series circuit, where the resistors are analogous to the boundary layers, and the voltage is analogous to the externally applied velocity difference between inner and outer cylinders.

The smooth boundaries are poor conductors (or large resistors) for angular momentum relative to rough walls. In this analogy, it is clear that the larger resistance dominates the total resistance in a series circuit.

The implication is that the smooth wall sustains a relatively large angular momentum gradient while only communicating a relatively small torque. Those cases with one rough and one smooth wall should therefore have the fluid coupling strongly to the rough wall whether it is rotating or not. The angular velocity (say at mid gap) would be biased toward the rough wall relative to the smooth-smooth case. Although we did not observe the mean angular velocity in this experiment, this hypothesis could be tested in future experiments.

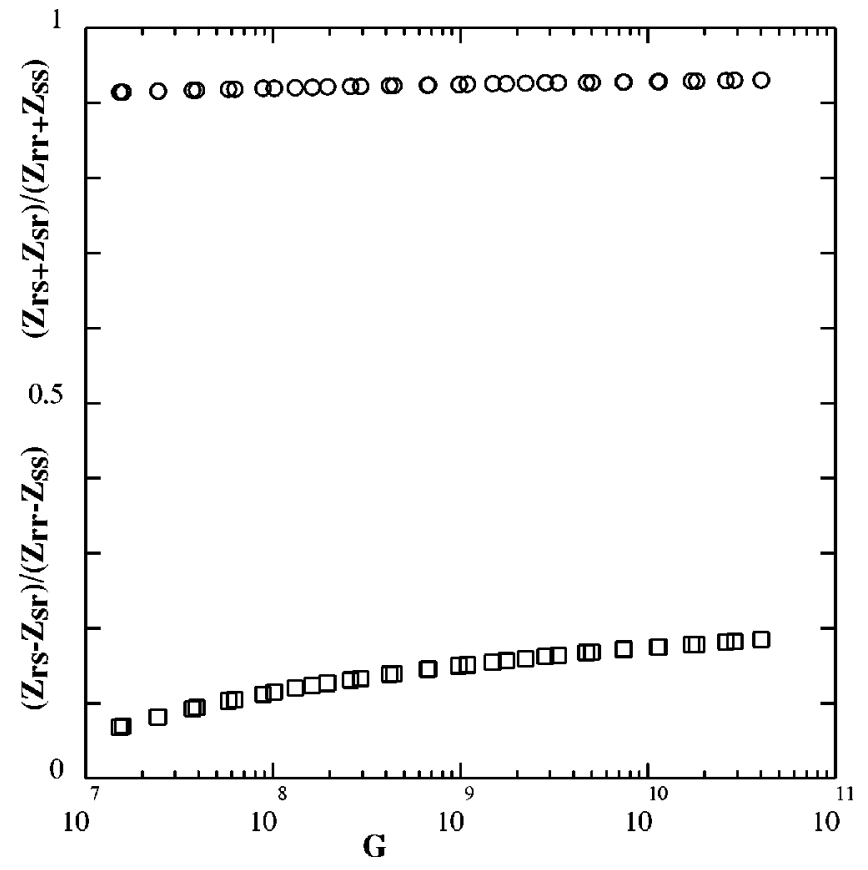

FIG. 4. A test of the impedance model for the boundary layers. $\left(Z_{r s}+Z_{s r}\right) /\left(Z_{r r}+Z_{s s}\right)$ and $\left(Z_{r s}-Z_{s r}\right) /\left(Z_{r r}-Z_{s s}\right)$ vs $G$. Both ratios would be one if the impedance model would exactly work. The data used for this test come from the fitted Prandtl-von Kármán forms shown in Fig. 3 (as data are needed at precisely the same Torques for each case).

The circuit analogy leads to a simple analytical model for the coupling of multiple boundaries in series. We propose a hydrodynamic Ohms' law

$$
Z(R) T=\Delta U
$$

where the velocity difference $\Delta U$ designates the (angular) momentum drop across the layer, $T$ the communicated torque, and $Z$ the impedance of the boundary layer. The impedance $Z$ depends not only on the Reynolds number but also on the nature, smooth or rough, of the wall. We then calculate the total velocity drop between cylinders as the drop across the inner boundary layer plus the drop across the outer boundary layer, ignoring any core region:

$$
\Delta U_{\text {in }}+\Delta U_{\text {out }}=\Delta U_{\text {total }}
$$

so that

$$
T Z_{\text {in }}(R)+T Z_{\text {out }}(R)=T Z_{\text {total }}(R) .
$$

The torques are equal between the two layers so we obtain $Z_{\text {total }}=Z_{\text {in }}+Z_{\text {out }}$ as in series resistances.

The characteristic impedances of the outer and inner boundaries would be different, even if both smooth or rough, due to geometric differences. Still, we can test this hypothesis of additive impedances by examining the following four cases:

$$
\begin{aligned}
& Z_{s s}=Z_{s, \text { inner }}+Z_{s, \text { outer }}, \\
& Z_{s r}=Z_{s, \text { inner }}+Z_{r, \text { outer }}, \\
& Z_{r s}=Z_{r, \text { inner }}+Z_{s, \text { outer }}
\end{aligned}
$$




$$
Z_{r r}=Z_{r, \text { inner }}+Z_{r, \text { outer }}
$$

These four equations result in the two expressions which contain measurable quantities only, namely,

$$
Z_{s s}+Z_{r r}=Z_{r s}+Z_{s r}
$$

and

$$
Z_{r r}-Z_{s s}=Z_{r s}-Z_{s r}
$$

These relationships indicate how the rough and smooth boundary layers may be combined in arbitrary series circuits of momentum transfer if the involved impedances are known. Note that expression (17) would even hold with the bulk contribution to the impedance in Eqs. (10)-(15), as this bulk contribution would cancel out.

We test the relationships in Eqs. (16) and (17) with the experimental data in Fig. 4. We plot the ratios of the lefthand side and the right-hand side of these equations. The ratio of the sums $\left(Z_{r s}+Z_{s r}\right) /\left(Z_{s s}+Z_{r r}\right)$ falls less than $10 \%$ below the theoretical expectation 1 over nearly three orders of magnitude in the Reynolds number. The residuals show a deviation diminishing at higher Reynolds numbers (smaller total impedances) possibly indicating that some communication between the boundary layers is not captured in this simple model. The ratio of the difference $\left(Z_{r s}-Z_{s r}\right) /\left(Z_{r r}\right.$ $\left.-Z_{s s}\right)$ is increasing with $\mathrm{Re}$, but still is about a factor of 5 smaller than 1 , showing quantitative shortcomings of our simplistic model.

Nevertheless, this circuit analogy could be used to make predictions for systems with different combinations of rough and smooth walls where the momentum must pass through a series of such boundary layers. One could even extend the model to make predictions about nested cylindrical systems, whose interior cylinders could rotate freely. How this model could be extended to systems with the momentum passing through heterogeneous boundary layers in parallel is uncertain — and would likely only be possible in quite simple geometric situations.

In conclusion, we have measured the Reynolds number dependence of the dimensionless torque $G$ in turbulent Taylor-Couette flow with rough and/or smooth walls. We have reported results for the four cases of two smooth walls, smooth-inner/rough-outer, rough-outer/smooth-inner, and two rough walls. The data are interpreted within the Grossmann-Lohse theory, strengthening the analogy between Taylor-Couette and Raleigh-Benard flows (also explored for turbulent flows in [29]). Perhaps surprisingly, we conclude that it is the smoothest wall that dominates the observed scalings, acting as the rate-limiting step for momentum transfer.

\section{ACKNOWLEDGMENTS}

The authors thank Harry Swinney (and the University of Texas) for loan of the apparatus used in the experiments, and G. Lewis, B.W. Zeff, W.L. Shew, D.D. Lanterman, and D. Martin for assistance. We would like to acknowledge K.R. Sreenivasan, S. Grossmann, F.H. Busse, and L.P. Kadanoff for helpful discussion during the early stages of this project. D.P.L. and C.R.D. acknowledge support by the National Science Foundation and the Research Corporation and T.H.v.d.B. and D.L. by FOM, which is financially supported by NWO, by the European Union (EU) through the European Research Network on "Nonideal Turbulence" (Contract No. HPRN-CT-200000162), and by the GermanIsraeli Foundation (GIF).
[1] L.N. Howard, J. Fluid Mech. 17, 405 (1963); C.R. Doering and P. Constantin, Phys. Rev. E 53, 5957 (1996).

[2] L.N. Howard, Annu. Rev. Fluid Mech. 4, 473 (1972); C.R. Doering and P. Constantin, Phys. Rev. E 49, 4087 (1994).

[3] Y. Shen, P. Tong, and K.Q. Xia, Phys. Rev. Lett. 76, 908 (1996).

[4] K.-Q. Xia and S.-L. Lui, Phys. Rev. Lett. 79, 5006 (1997).

[5] Y.B. Du and P. Tong, Phys. Rev. Lett. 81, 987 (1998).

[6] Y.B. Du and P. Tong, J. Fluid Mech. 407, 57 (2000).

[7] Y.B. Du and P. Tong, Phys. Rev. E 63, 046303 (2001).

[8] S. Ciliberto and C. Laroche, Phys. Rev. Lett. 82, 3998 (1999).

[9] P.E. Roche, B. Castaing, B. Chabaud, and B. Hebral, Phys. Rev. E 63, 045303 (2001).

[10] G. Ahlers and X. Xu, Phys. Rev. Lett. 86, 3320 (2001).

[11] K.-Q. Xia, S. Lam, and S.Q. Zhou, Phys. Rev. Lett. 88, 064501 (2002).

[12] R. Verzicco and R. Camussi, J. Fluid Mech. 383, 55 (1999).

[13] L.D. Landau and E.M. Lifshitz, Fluid Mechanics (Pergamon Press, Oxford, 1987).

[14] S.B. Pope, Turbulent Flow (Cambridge University Press, Cambridge, 2000).
[15] O. Cadot et al., Phys. Rev. E 56, 427 (1997).

[16] U. Frisch, Turbulence (Cambridge University Press, Cambridge, 1995).

[17] D.P. Lathrop, J. Fineberg, and H.L. Swinney, Phys. Rev. Lett. 68, 1515 (1992).

[18] D.P. Lathrop, J. Fineberg, and H.L. Swinney, Phys. Rev. A 46, 6390 (1992).

[19] G.S. Lewis and H.L. Swinney, Phys. Rev. E 59, 5457 (1999).

[20] C.R. Doering and P. Constantin, Phys. Rev. Lett. 69, 1648 (1992).

[21] P. Constantin and C. R. Doering (unpublished).

[22] S. Grossmann and D. Lohse, J. Fluid Mech. 407, 27 (2000).

[23] S. Grossmann and D. Lohse, Phys. Rev. Lett. 86, 3316 (2001).

[24] S. Grossmann and D. Lohse, Phys. Rev. E 66, 016305 (2002).

[25] B. Eckhardt, S. Grossmann, and D. Lohse, Eur. Phys. J. B 18, 541 (2000).

[26] R.H. Kraichnan, Phys. Fluids 5, 1374 (1962).

[27] E.A. Spiegel, Annu. Rev. Astron. Astrophys. 9, 323 (1971).

[28] D. Lohse and F. Toschi, Phys. Rev. Lett. 90, 034502 (2003).

[29] B. Dubrulle and F. Hersant, Eur. Phys. J. B 26, 379 (2002). 